\title{
Facile synthesis of hierarchical triazine-based porous carbons for hydrogen storage
}

Xin-Ming Hu, ${ }^{a, b}$ Qi Chen, ${ }^{a}$ Yan-Chao Zhao, ${ }^{a}$ Bo W. Laursen, ${ }^{b}$ Bao-Hang $\operatorname{Han} * a$

${ }^{a}$ CAS Key Laboratory of Nanosystem and Hierarchical Fabrication, National Center for Nanoscience and Technology, Beijing 100190, China Tel: +86108254 5576. Email: hanbh@nanoctr.cn

${ }^{b}$ Nano-Science Center and Department of Chemistry, University of Copenhagen, Universitetsparken 5, DK-2100 Kфbenhavn Ø, Denmark 


\begin{abstract}
Triazine-based porous carbon materials (TPCs) have been synthesized via cyclotrimerization of aromatic tetranitriles and in situ carbonization. The resulting TPCs have high surface area (above $1200 \mathrm{~m}^{2} \mathrm{~g}^{-1}$ ), large pore volume (above $1.4 \mathrm{~cm}^{3} \mathrm{~g}^{-1}$ ), and hierarchical pore structures with micropores $(0.63-1.24 \mathrm{~nm})$ and mesopores $(2.4-20$ $\mathrm{nm})$. Gas adsorption experiments demonstrate their promising hydrogen uptake capacity, up to $2.34 \mathrm{wt} \%$ at $77 \mathrm{~K}$ and $1.0 \mathrm{bar}$, due to the hierarchical porosity that facilitates the diffusion and adsorption of gas molecules.
\end{abstract}

Keywords: Cyclotrimerization of nitrile; Carbonization; Triazine-based porous carbon; Hierarchical; Hydrogen uptake. 


\section{Introduction}

The development of efficient and safe methods for hydrogen storage is still an essential prerequisite for utilization of hydrogen energy. One conventional approach is to chemisorb hydrogen as metal or chemical hydrides, which has been comprehensively investigated and proved fairly efficient $[1,2]$. However, the high discharge temperature and poor cycle performance remain the bottleneck for their practical applications [2]. An alternative approach to store hydrogen is through physisorption in porous media, which has advantages of fast kinetics and complete reversibility [3]. Of various porous materials, porous carbons are of particular interest thanks to their easy availability and excellent stability.

A simple approach to achieve high hydrogen uptake in porous carbons is to increase the specific surface area [4]. Particular efforts have also been devoted to enhancing hydrogen uptake by creation of micropores, and ultramicropores $(<0.7 \mathrm{~nm})$ in particular, where improved interactions between carbon and hydrogen molecules are present $[3,5]$. This approach seems more essential as micropores contribute dominantly to the high surface area and are most suitable for trapping of small gas molecules. On the other hand, purely microporous materials may have some limitations due to low permeability and restricted access to active sites for hydrogen adsorption. Mesopores, though they do not adsorb hydrogen efficiently, facilitate hydrogen transport in the porous media. To achieve fast and efficient hydrogen storage, an interconnected porous architecture with both micro- and mesoporosity (hierarchical structure) is thus essential. 
To create hierarchical porous carbons, sacrificial-template-based method is typically employed [6,7]. This method requires introduction and removal of templates and seems somewhat complicated and cost-ineffective. Thus, simple synthesis strategies are still highly desirable. Cyclotrimerization of aromatic nitriles has been proved highly efficient for construction of porous covalent triazine-based frameworks (CTFs) [8]. In such reaction, zinc chloride acts as both catalyst and solvent. Note that zinc chloride has also been widely used as activating reagents for synthesis of various carbon materials $[9,10]$. Pushing the nitrile cyclotrimerization reaction further to the carbonization stage is expected to produce carbon materials with both microporosity and mesoporosity. A systematical study by Kuhn and co-workers has demonstrated that cyclotrimerization of nitriles in zinc chloride at $400{ }^{\circ} \mathrm{C}$ produces microporous polymers and that higher reaction temperatures $\left(>600{ }^{\circ} \mathrm{C}\right)$ induce the formation of additional mesopores via carbonization [11]. Their subsequent work indicates that more equivalents of zinc chloride can also induce the carbonization (at $400{ }^{\circ} \mathrm{C}$ ) and lead to the formation of mesopores, though micropores are still dominant in the final materials $[12,13]$.

Recently, we introduced a combined cyclotrimerization and carbonization process that allows the formation of a nitrogen-rich porous carbon (TPC-1) from a perfluorinated aromatic nitriles [14]. TPC-1 exhibits dominant micropores, though mesopores are present as well [14]. By use of different precursors and increasing amount of zinc chloride, triazine-based porous carbons (TPCs) with varying porosities and chemical compositions could be expected. Herein, we report two more TPCs that 
are prepared by combined cyclotrimerization and carbonization of two aromatic tetranitriles in twenty equivalents of zinc chloride. Both TPCs prove to be nitrogen-free and feature well-defined bimodal pore structure, thereby exhibiting exceptionally high hydrogen uptake capacities.

\section{Experimental}

\subsection{Materials}

All chemicals were purchased from commercial suppliers and used without further purification unless otherwise stated. Anhydrous zinc chloride was further dried at $120{ }^{\circ} \mathrm{C}$ in vacuo overnight prior to use. Tetra(4-bromophenyl)methane [15] and tetra(4-bromophenyl)ethene [16] were synthesized according to reported procedures. Ultra-high-purity grade gases were used for all gas adsorption experiments.

\subsection{Synthesis of monomers}

Tetra(4-cyanobiphenyl)methane (M-2). To a round-bottom flask were added tetra(4-bromophenyl)methane (1.0 g, $1.57 \mathrm{mmol})$, 4-cyanophenylboronic acid (1.39 g, $9.43 \mathrm{mmol})$, potassium carbonate $(4.35 \mathrm{~g}, 31.5 \mathrm{mmol})$, and $N, N$-dimethylformamide (30 mL). The mixture was degassed by the freeze-pump-thaw cycles. Tetrakis(triphenylphosphine)palladium $(80 \mathrm{mg}$ ) was then added and the formed mixture was heated at $100{ }^{\circ} \mathrm{C}$ under nitrogen atmosphere for $24 \mathrm{~h}$. The reaction mixture was cooled down to room temperature and poured into deionized water with stirring. The precipitate was dissolved in dichloromethane and washed with deionized water and brine. The organic phase was collected and the solvent was evaporated. The residue was 
purified by column chromatography on silica gel (dichloromethane/methanol, $\mathrm{v} / \mathrm{v}=$ 100:1) to afford a white solid $(0.51 \mathrm{~g}, 45 \%) .{ }^{1} \mathrm{H}$ NMR $\left(400 \mathrm{MHz}, \mathrm{CDCl}_{3}\right): \delta$ (ppm) 7.74-7.69 (m, 16H), $7.57(\mathrm{~d}, J=8.0 \mathrm{~Hz}, 8 \mathrm{H}), 7.44(\mathrm{~d}, J=8.0 \mathrm{~Hz}, 8 \mathrm{H}) .{ }^{13} \mathrm{C}$ NMR $(100$ $\left.\mathrm{MHz}, \mathrm{CDCl}_{3}\right): \delta(\mathrm{ppm}) 146.7,144.9,137.2,132.8,131.74,127.7,126.84,119.0,111.3$, 64.6. MS (MALDI-TOF) m/z: calcd for $\mathrm{C}_{53} \mathrm{H}_{32} \mathrm{~N}_{4} 724.26$ [M], found 724.21.

Similarly, tetra(4-cyanobiphenyl)ethene (M-3) was synthesized from tetra(4-bromophenyl)ethene. Purification by column chromatography on silica gel (dichloromethane/methanol, v/v $=150: 1)$ afforded a yellow solid $(0.64 \mathrm{~g}, 56 \%) .{ }^{1} \mathrm{H}$ NMR (400 MHz, $\left.\mathrm{CDCl}_{3}\right): \delta(\mathrm{ppm}) 7.71-7.64(\mathrm{~m}, 16 \mathrm{H}), 7.42(\mathrm{~d}, J=8.0 \mathrm{~Hz}, 8 \mathrm{H}), 7.22$ $(\mathrm{d}, J=8.0 \mathrm{~Hz}, 8 \mathrm{H}) .{ }^{13} \mathrm{C} \mathrm{NMR}\left(100 \mathrm{MHz}, \mathrm{CDCl}_{3}\right): \delta(\mathrm{ppm}) 144.9,143.8,140.8,137.6$, 132.7, 132.3, 127.6, 126.9, 119.0, 111.1. MS (MALDI-TOF) m/z: calcd for $\mathrm{C}_{54} \mathrm{H}_{32} \mathrm{~N}_{4}$ $736.26[\mathrm{M}]$, found 736.66 .

\subsection{Preparation of TPCs}

TPC-2. To a glass ampoule were added tetra(4-cyanobiphenyl)methane (M-2, 150 $\mathrm{mg}, 0.21 \mathrm{mmol}$ ) and anhydrous zinc chloride $(564 \mathrm{mg}, 4.14 \mathrm{mmol})$ under nitrogen atmosphere. The ampoule was evacuated, sealed, and heated to $400{ }^{\circ} \mathrm{C}$ over $1 \mathrm{~h}$. After keeping at this temperature for $40 \mathrm{~h}$, the ampoule was cooled down to room temperature. The reaction mixture was washed thoroughly with water and diluted aqueous hydrochloric acid solution to remove zinc chloride. The resulting black product was further purified by Soxhlet extraction with water, tetrahydrofuran, and dichloromethane. After drying in vacuo at $150{ }^{\circ} \mathrm{C}, 125 \mathrm{mg}$ of black powder was obtained, corresponding 
to $83 \%$ of the starting material by mass.

Similarly, TPC-3 was prepared from M-3 in 75\% yield.

\subsection{Characterization and measurement}

${ }^{1} \mathrm{H}$ and ${ }^{13} \mathrm{C}$ NMR spectra were recorded on a Bruker Avance DMX-400 NMR spectrometer (Bruker, Germany) using the residual solvent as internal reference. MALDI-TOF mass spectra were recorded on a Bruker Autoflex vertical reflector instrument (Bruker, Germany). IR spectra were measured on a Bruker ALPHA FT-IR spectrometer (Bruker, Germany) with an attenuated total reflection accessory. Field emission scanning electron microscopy (SEM) observations were carried out on a JSM-6320F scanning electron microscope (JEOL, Japan) at an accelerating voltage of $10 \mathrm{kV}$. The powder of the as-prepared materials was placed on a silica wafer before measurement. Raman spectroscopy, X-ray diffraction (XRD), thermogravimetric analysis (TGA), X-ray photoelectron spectroscopy (XPS), and gas adsorption experiments were performed according to previously reported procedures [14].

\section{Results and discussion}

\subsection{Synthesis and characterization of TPCs}

The synthesis routes to TPCs are presented in Scheme 1. The two aromatic tetranitrile monomers (M-2 and M-3) are synthesized via fourfold palladium-catalyzed Suzuki coupling reaction between tetra(4-bromophenyl)methane/-ethene and 4-cyanophenylboronic acid. Heating the mixture of the monomer and zinc chloride in a sealed glass ampoule at $400{ }^{\circ} \mathrm{C}$ produces the desired porous carbon materials (TPC-2 
and TPC-3, from M-2 and M-3, respectively). Note that Ren et al. [17] and Bhunia et al. [18] have reported respectively porous frameworks made from tetra(4-cyanophenyl)methane/-ethene, similar tetranitriles to ours. In their reports the final materials prepared in ten equivalents of or less zinc chloride are claimed to be either porous aromatic frameworks (PAF-2) or CTFs (PCTF-1), rather than the desired TPCs. Thus, to ensure the efficient carbonization of the resulting polytriazine networks, twenty equivalents of zinc chloride are used. Analogous to the synthesis of CTFs, the reaction is carried out at autogenic pressure and no apparent pressure is detected when the ampoule is opened [8]. Black monolith that can be grounded to powder is obtained in the yield of ca. $80 \%$ by successive washing away the salt from the final mixture with aqueous hydrochloric acid solution and deionized water followed by Soxhlet extraction. It is noteworthy that overpressure is detected and the yield is only $54 \%$ for the synthesis of TPC-1 [14], implying that the carbonization process for TPC-2/-3 and TPC-1 occurs through different pathways that will be discussed after structural and porosity characterization.

The as-prepared TPCs were characterized by IR spectroscopy (Figure 1a). The IR spectra of both monomers exhibit intense carbonitrile band around $2220 \mathrm{~cm}^{-1}$, which is not detected anymore for TPCs. The disappearance of such band indicates the complete transformation of the nitriles. Unlike the synthesis of CTFs, the triazine vibration band around $1360 \mathrm{~cm}^{-1}$ is not observed [8,18,19]. More significantly, no obvious vibration bands can be detected for the TPCs. Even the original strong bands between 1490 and 
$1600 \mathrm{~cm}^{-1}$ that are assigned to skeleton vibration of the aromatic rings in monomers are extinguished. The most likely reason for the unusual flattening of the IR spectra is that complete carbonization occurs during the reaction.

Considering the possible pure carbon composition of the final materials, implied by the IR analysis, Raman spectroscopy was applied to characterize the resulting materials. As shown in the Raman spectra (Figure 1b), the Raman shift around 2225 $\mathrm{cm}^{-1}$ that belongs to cyano group disappears completely, in agreement with the results from IR analysis. Furthermore, both TPCs exhibit two broad peaks centered at 1335 and $1589 \mathrm{~cm}^{-1}$, corresponding to $D$ - and $G$ - Raman bands of amorphous graphitic carbon, respectively. In general, the $D$-band represents the presence of disordered carbon structures, while the $G$-band is related to the vibration of $s p^{2}$ bonded carbon atoms in a two-dimensional hexagonal lattice. The locations of these two peaks resemble those typically observed in graphitized carbon materials [14,20,21], indicative of the graphitization of TPCs to some degree. The $D / G$ ratios for TPC-2 and TPC-3 are calculated to be 0.88 and 0.85 , respectively, smaller than that (> 1.0) of TPC-1 [14] and some other nitrogen-doping porous carbons [20,21], which indicates the higher degree of graphitization in the as-prepared TPCs. The close $D / G$ ratios for TPC-2 and TPC-3 suggest that the two TPCs have a similar degree of carbonization, in spite of the differences in their monomer structures. Accordingly, TPCs can be regarded as partially graphitic carbon materials consisting of both small graphitic sheets and amorphous carbon. 
XPS analysis confirms that the two TPCs are composed of carbon. The XPS survey spectra (Figure 2a) of both TPCs exhibit a dominant peak centered at $285.1 \mathrm{eV}$ and a quite weak peak at $533.1 \mathrm{eV}$, corresponding to $\mathrm{C} 1 \mathrm{~s}$ and $\mathrm{O} 1 \mathrm{~s}$, respectively. The observed oxygen signal is believed to come from the adsorbed oxygen and moisture [22]. The C 1s spectra (Figure 2b and 2c) of both TPCs can be well-fitted by one peak, related to the graphitic $s p^{2}$ carbon, which at first glance seems contradictory to the results of Raman spectra (Figure 1b) where amorphous carbon signal are clearly shown. A recent calculation for the defect percentage of graphene conducted by Bjerglund et al. reveals that approximate one out of four hundred carbon atoms in graphene is functionalized when $D / G$ ratio reaches 1.0 [23], which indicates the agreement between Raman spectra and XPS analysis and makes us more convinced that a certain degree of graphitization are accomplished for TPC-2 and TPC-3. This point is also supported by the powder XRD patterns (Figure S1, Supplementary Data), which show two broad peaks at $2 \theta \approx 24$ and $44^{\circ}$, though the latter is quite weak. These two peaks correspond to the carbon (002) and (101) diffractions, as typical characteristic peaks of graphitic carbon [20]. On the other hand, there is no detectable nitrogen signal (expected at ca. $399 \mathrm{eV})$, which discloses the complete nitrogen elimination during the polymerization/carbonization process. At the selected reaction temperature $\left(400{ }^{\circ} \mathrm{C}\right)$, the complete depletion of nitrogen is unusual, though decreased nitrogen content in reported CTFs is observed $[8,11,12,18]$. The complete nitrogen depletion could be explained by the use of more equivalents of zinc chloride (20 equiv.) compared to the 
synthesis of CTFs (typically 10 equiv. or less) [17,18]. It has been demonstrated that more severe nitrogen depletion takes place with increasing amount of salt [12]. XPS survey spectrum confirms the complete removal of the salt, as no $\mathrm{Zn}(\mathrm{ca} .1020 \mathrm{eV}$ ) or $\mathrm{Cl}$ (ca. $200 \mathrm{eV}$ ) signal from zinc chloride is detected. Thus, it can be concluded that the final products we obtained are nitrogen-free carbon materials, rather than the previously reported CTFs obtained from related nitriles. SEM images reveal that both TPCs are made up of uniform particles (Figure S2, Supplementary Data), in particular, TPC-3 consists of microspheres with diameter around $50 \mathrm{~nm}$. Thermogravimetric analysis demonstrates the good stability of both TPCs, less than $20 \%$ weight is lost when the temperature is raised to $800{ }^{\circ} \mathrm{C}$ under nitrogen atmosphere (Figure S3, Supplementary Data).

\subsection{Porosity and hydrogen storage}

Nitrogen sorption measurement was conducted to evaluate the porosities of the two TPCs. Figure 3 a presents the nitrogen adsorption-desorption isotherms measured at $77 \mathrm{~K}$. Both isotherms are of type IV according to IUPAC classification [24]. A steep rise in nitrogen uptake at very low relative pressures $\left(P / P_{0}<0.01\right)$ indicates the presence of micropores. At relative pressures higher than 0.10 , no adsorption plateau is observed but a gradual increase. Moreover, the pronounced hysteresis loops in the relative pressure range of $0.5-0.8$ indicate the presence of well-defined mesopores in both TPCs. In contrast, reported PAF-2 and PCTF-1, derived from monomers with similar structure to TPC-2 and TPC-3, exhibit negligible hysteresis in their nitrogen physisorption 
isotherms $[17,18]$. The BET specific surface area results of TPC-2 and TPC-3 are calculated to be 1250 and $1530 \mathrm{~m}^{2} \mathrm{~g}^{-1}$, respectively, which are moderate among CTFs and other porous carbon materials (Table S1, Supplementary Data). The pore volumes estimated at $P / P_{0}=0.97$ are 1.45 and $1.57 \mathrm{~cm}^{3} \mathrm{~g}^{-1}$, respectively, close to that of PCTF-1 $\left(1.56 \mathrm{~cm}^{3} \mathrm{~g}^{-1}\right)$, and higher than that of TPC-1 $\left(1.23 \mathrm{~cm}^{3} \mathrm{~g}^{-1}\right)$, though PCTF-1 and TPC-1 show much higher surface area [18]. The relatively low BET specific surface area but similar or higher pore volume value further indicates the presence of massive mesopores in TPC-2 and TPC-3.

The pore size distribution (PSD) profile was analyzed using nonlocal density functional theory (NLDFT) model (Figure 3b). Both TPCs exhibit bimodal PSD, with micropore diameter in the range of $0.63-1.24 \mathrm{~nm}$ and mesopore diameter in the range of 2.4-20 nm. Cyclotrimerization of nitriles is expected to result in the formation of CTFs, which typically exhibit microporosity $[8,17,19]$. The distinct and broad range of mesopores are assumed to arise from the decomposition of triazine rings and subsequent $[\mathrm{CN}]$-elimination induced by more equivalents of zinc chloride. In other words, the depletion of nitrogen occurs via the sequential formation and decomposition of triazine rings, rather than the direct loss of $[\mathrm{CN}]$ group at the beginning. This assumption can be supported by the following points: (1) triazine rings can be easily form under the selected conditions [8]; (2) a cyano-free precursor (biphenyl) is only able to produce a small amount of non-porous carbon under similar conditions [12]; (3) the formation of $\mathrm{CN}$ and $\mathrm{HCN}$ is observed by Bhunia et al. through thermogravimetry-mass 
spectrometry coupled analysis [18]. This proposed process for the formation of TPC-2 and TPC-3 is different from that of TPC-1, where defluorination occurs [14]. The detailed porosity parameters of TPCs, PAF-2, and PCTF-1 are included in Table 1. The level of microporosity in TPCs is assessed by the ratio of micropore volume to total pore volume $\left(V_{\text {micro }} / V_{\text {total }}\right)$. The pore volume at $P / P_{0}=0.1$ is approximately regarded as the micropore volume for both TPCs, since micropore filling finishes at this relative pressure. Both TPCs show small $V_{\text {micro }} / V_{\text {total }}$ values $(0.31$ and 0.34$)$, lower than the typical value $(>0.5)$ of reported CTFs $[18,19,25]$. This confirms that mesopores are dominant in the as-prepared TPCs, in contrast to PAF-2, PCTF-1, and other CTFs.

Since Kuhn et al. pioneered CTFs in 2008 [8], continuous effort has been devoted to the synthesis of various CTFs $[17,18,19,25,26,27,28,29,30]$. In principle, all CTFs are considered as a type of porous organic polymers in terms of their compositions and structures. It has to point out that a sign of carbonization, that is, reduction of nitrogen content in the final materials, has been observed by many groups $[8,11,12,18,25,29]$, where partially carbonized CTFs are obtained indeed in the presence of ten equivalents of or less zinc chloride. Furthermore, for most CTFs micropores are predominant $[17,18,19,26,27]$. In the present work, in addition to cyclotrimerization of nitriles, complete carbonization is accomplished in twenty equivalents of zinc chloride, leading to the formation of nitrogen-free, graphitic, and hierarchical carbon with substantial mesopores.

To assess their potential for storage of energy gases, hydrogen adsorption 
isotherms of both TPCs at low pressure were collected (Figure 4). TPC-2 and TPC-3 adsorb 2.34 and $1.84 \mathrm{wt} \%$ of hydrogen at $77 \mathrm{~K}$ and 1.0 bar. To the best of our knowledge, the hydrogen uptake capacity of TPC-2 is higher than CTFs and comparable to other porous carbon materials, as shown in Table S1 (Supplementary Data). The exceptionally high hydrogen uptake is attributed to the simultaneous presence of (ultra)micropores that are suitable for hydrogen adsorption and mesopores that are favorable for gas transportation, as shown in Figure 3b. It can further be found that the hydrogen uptake capacities for porous carbon materials are generally higher than polymeric CTFs, which provides an implication that carbonization might be able to produce porosities that are more suitable for hydrogen trapping. Thus, further carbonization of CTFs to carbon materials is beneficial to hydrogen uptake.

\section{Conclusions}

A simple one-pot procedure for synthesis of graphitic porous carbons has been developed. Cyclotrimerization of nitriles and in situ carbonization lead to the formation of TPCs, rather than the widely reported CTFs. In particular, TPC-2 has a BET specific high surface area, large pore volume, and hierarchical pore structures. These features make TPCs promising for hydrogen storage. We believe that the present method can be extended to a wide variety of nitrile precursors.

\section{Acknowledgements}

This work was supported by Chinese-Danish Center for Molecular 
Nanoelectronics funded by the National Science Foundation of China (No. 61261130092) and the Danish National Research Foundation. The financial support of the National Science Foundation of China (Grant no. 21374024) and the Ministry of Science and Technology of China (Grant 2014CB932204) is also acknowledged.

\section{References}

[1] S.-I. Orimo, Y. Nakamori, J. R. Eliseo, A. Züttel, C. M. Jensen, Chem. Rev. 107 (2007), 4111-4132.

[2] M. Felderhoff, C. Weidenthaler, R. von Helmolt, U. Eberle, Phys. Chem. Chem. Phys. 9 (2007) 2643-2653.

[3] A. W. C. van den Berg, C. O. Areán, Chem. Commun. (2008) 668-681.

[4] B. Panella, M. Hirscher, S. Roth, Carbon 43 (2005) 2209-2214.

[5] G. Yushin, R. Dash, J. Jagiello, J. E. Fischer, Y. Gogotsi, Adv. Funct. Mater. 16 (2006) $2288-2293$.

[6] B. Liu, H. Shioyama, T. Akita, Q. Xu, J. Am. Chem. Soc. 130 (2008) 5390-5391.

[7] M. J. Valero-Romero, E. M. Márquez-Franco, J. Bedia, J. Rodríguez-Mirasol, T. Cordero, Micropor. Mesopor. Mater. 196 (2014) 68-78.

[8] P. Kuhn, M. Antonietti, A. Thomas, Angew. Chem. Int. Ed. 47 (2008) 3450-3453.

[9] S. Yorgun, N. Vural, H. Demiral, Micropor. Mesopor. Mater. 122 (2009) 189-194.

[10] T. H. Wang, S. X. Tan, C. H. Liang, Carbon 47 (2009) 1880-1883.

[11] P. Kuhn, A. Forget, D. S. Su, A. Thomas, M. Antonietti, J. Am. Chem. Soc. 130 
(2008) 13333-13337.

[12] P. Kuhn, A. Forget, J. Hartmann, A. Thomas, M. Antonietti, Adv. Mater. 21 (2009) 897-901.

[13] P. Kuhn, A. Thomas, M. Antonietti, Macromolecules 42 (2009) 319-326.

[14] X.-M. Hu, Q. Chen, Y.-C. Zhao, B. W. Laursen, B.-H. Han, J. Mater. Chem. A 2 (2014) 14201-14208.

[15] M. Grimm, B. Kirste, H. Kurreck, Angew. Chem. Int. Ed. 25 (1986) 1097-1098.

[16] Z. Chang, Y. Jiang, B. He, J. Chen, Z. Yang, P. Lu, H. S. Kwok, Z. Zhao, H. Qiu, B. Z. Tang, Chem. Commun. 49 (2013) 594-596.

[17] H. Ren, T. Ben, E. S. Wang, X. F. Jing, M. Xue, B. B. Liu, Y. Cui, S. L. Qiu, G. S. Zhu, Chem. Commun. 46 (2010) 291-293.

[18] A. Bhunia, V. Vasylyeva, C. Janiak, Chem. Commun. 49 (2013) 3961-3963.

[19] S. J. Ren, M. J. Bojdys, R. Dawson, A. Laybourn, Y. Z. Khimyak, D. J. Adams, A. I. Cooper, Adv. Mater. 24 (2012) 2357-2361

[20] Q. F. Wang, W. Xia, W. H. Guo, L. An, D. G. Xia, R. Q. Zou, Chem. Asian J. 8 (2013) 1879-1885.

[21] Y. Mao, H. Duan, B. Xu, L. Zhang, Y. S. Hu, C. C. Zhao, Z. X. Wang, L. Q. Chen, Y. S. Yang, Energy Environ. Sci. 5 (2012) 7950-7955.

[22] B. Marchon, J. Carrazza, H. Heinemann, G. A. Somorjai, Carbon 26 (1988) $507-514$

[23] E. Bjerglund, M. Kongsfelt, K. Shimizu, B. B. E. Jensen, L. Koefoed, M. Ceccato, 
T. Skrydstrup, S. U. Pedersen, K. Daasbjerg, Langmuir 30 (2014) 6622-6628.

[24] K. S. W. Sing, D. H. Everett, R. A. W. Haul, L. Moscou, R. A. Pierotti, J. Rouquerol, T. Siemieniewska, Pure Appl. Chem. 57 (1985) 603-619.

[25] A. Bhunia, I. Boldog, A. Moller, C. Janiak, J. Mater. Chem. A 1 (2013) 14990-14999.

[26] X. M. Liu, H. Li, Y. W. Zhang, B. Xu, A. Sigen, H. Xia, Y. Mu, Polym. Chem. 4 (2013) 2445-2448.

[27] Y. F. Zhao, K. X. Yao, B. Y. Teng, T. Zhang, Y. Han, Energy Environ. Sci. 6 (2013) $3684-3692$.

[28] S. Hug, M. B. Mesch, H. Oh, N. Popp, M. Hirscher, J. Senker, B. V. Lotsch, J. Mater. Chem. A 2 (2014) 5928-5936.

[29] Y. Liu, S. Wu, G. Wang, G. Yu, J. Guan, C. Pan, Z. Wang, J. Mater. Chem. A 2 (2014) 7795-7801.

[30] S. Wu, Y. Liu, G. Yu, J. Guan, C. Pan, Y. Du, X. Xiong, Z. Wang, Macromolecules 47 (2014) 2875-2882.

[31] G. P. Hao, W. C. Li, D. Qian, G. H. Wang, W. P. Zhang, T. Zhang, A. Q. Wang, F. Schuth, H. J. Bongard, A. H. Lu, J. Am. Chem. Soc. 133 (2011), 11378-11388. 
Table 1. Porosity parameters of TPCs and a comparison with reported PAF-2 and PCTF-1.

\begin{tabular}{ccccccc}
\hline Material & $\begin{array}{c}S_{\text {BET }} \\
\left(\mathrm{m}^{2} \mathrm{~g}^{-1}\right)^{a}\end{array}$ & $\begin{array}{c}V_{\text {total }} \\
\left(\mathrm{cm}^{3} \mathrm{~g}^{-1}\right)^{b}\end{array}$ & $\begin{array}{c}V_{\text {micro }} \\
\left(\mathrm{cm}^{3} \mathrm{~g}^{-1}\right)^{c}\end{array}$ & $V_{\text {micro }} / V_{\text {total }}$ & $\begin{array}{c}D_{\text {pore }} \\
(\mathrm{nm})^{d}\end{array}$ & References \\
\hline TPC-2 & 1250 & 1.45 & 0.45 & 0.31 & $\begin{array}{c}0.63-1.24 \\
2.34-20\end{array}$ & This work \\
TPC-3 & 1530 & 1.57 & 0.54 & 0.34 & $\begin{array}{c}0.63-1.24 \\
2.45-20\end{array}$ & This work \\
PAF-2 & 891 & - & - & - & 1.06 & 17 \\
PCTF-1 & 2235 & $1.56^{e}$ & 0.79 & 0.51 & $<1,2-6$ & 18 \\
\hline
\end{tabular}

${ }^{a}$ The specific surface area calculated from the nitrogen adsorption isotherm using the BET method at the relative pressure range of $0.05-0.2{ }^{b}$ Total pore volume at $P / P_{0}=$ 0.97. ${ }^{c}$ Micropore volume estimated at $P / P_{0}=0.10 .{ }^{d}$ Dominant pore size determined by NLDFT method. ${ }^{e}$ Total pore volume at $P / P_{0}=0.95$. "-“ denotes that no data is available. 


\section{Figure captions}

Scheme 1. Synthesis of TPCs.

Figure 1. IR (a) and Raman spectra (b) of the monomers and TPCs.

Figure 2. XPS spectra of TPCs. (a) Survey, (b) C 1s of TPC-2 and (c) TPC-3.

Figure 3. (a) Nitrogen adsorption-desorption isotherms measured at $77 \mathrm{~K}$ (the adsorption and desorption isotherms are labeled with solid and open symbols, respectively); (b) pore size distribution profiles calculated by the NLDFT method.

Figure 4. Hydrogen adsorption isotherms at $77 \mathrm{~K}$. 

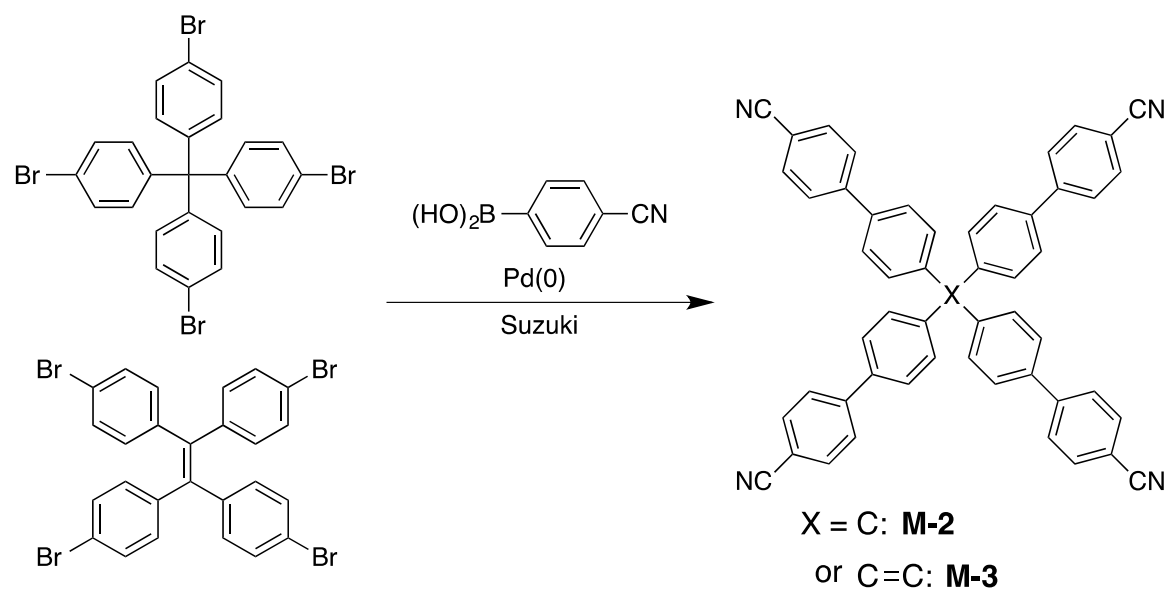

$$
X=C: M-2
$$

or $\mathrm{C}=\mathrm{C}: \mathbf{M}-\mathbf{3}$

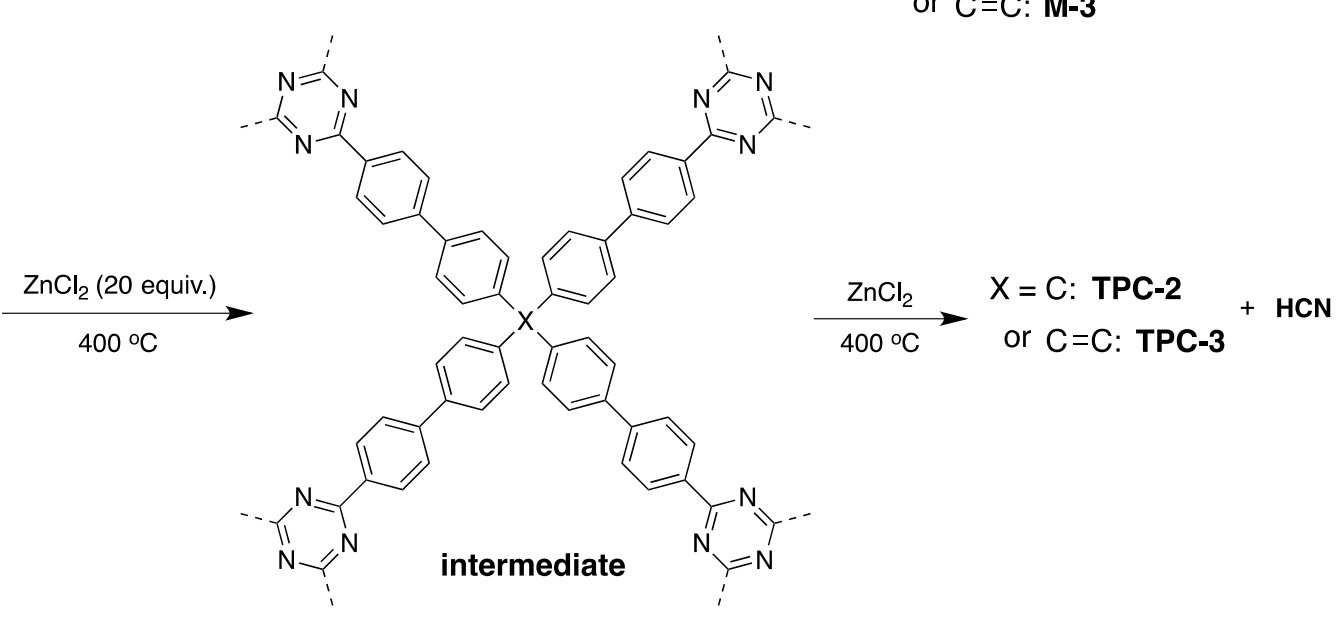

Scheme 1 

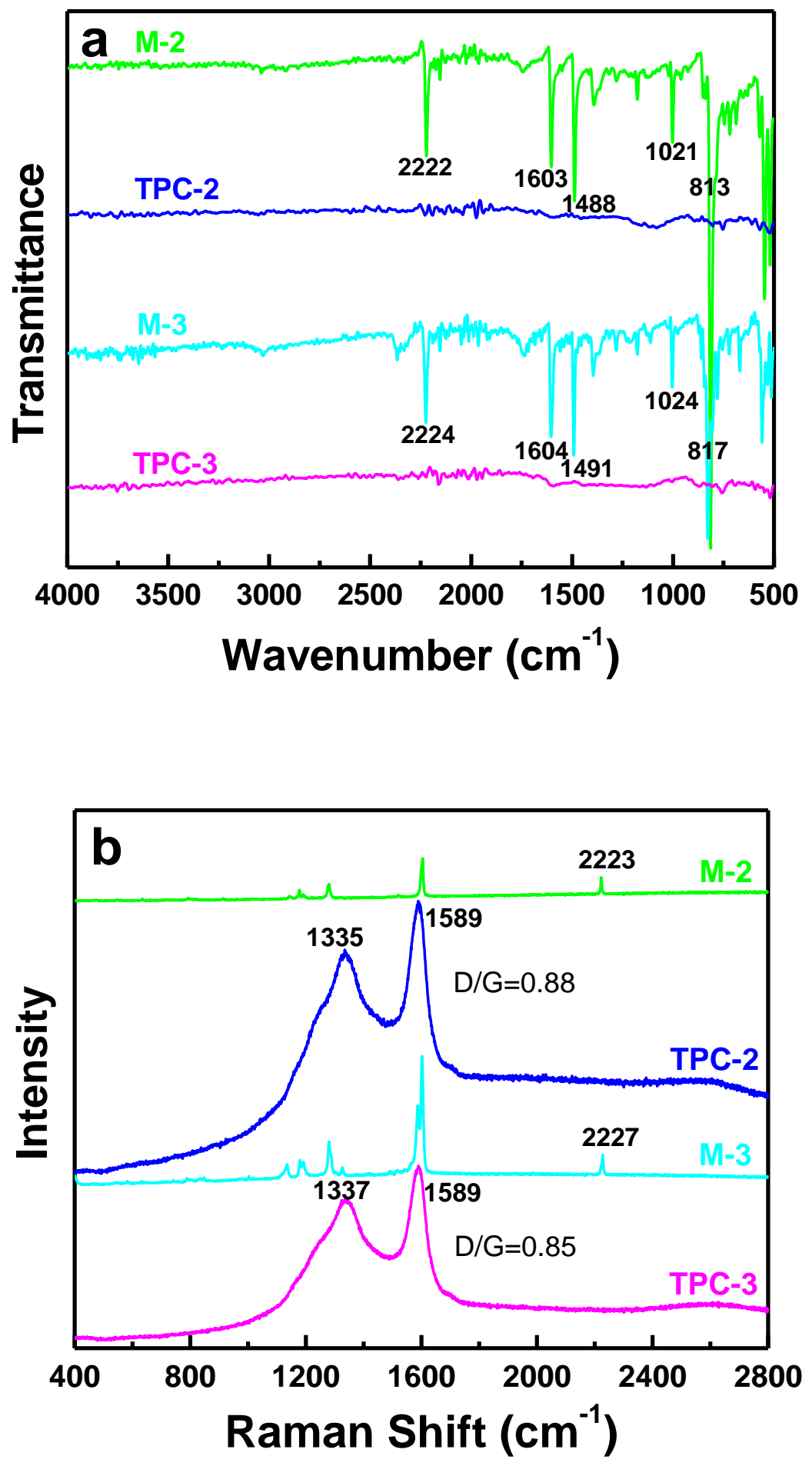

Figure 1 

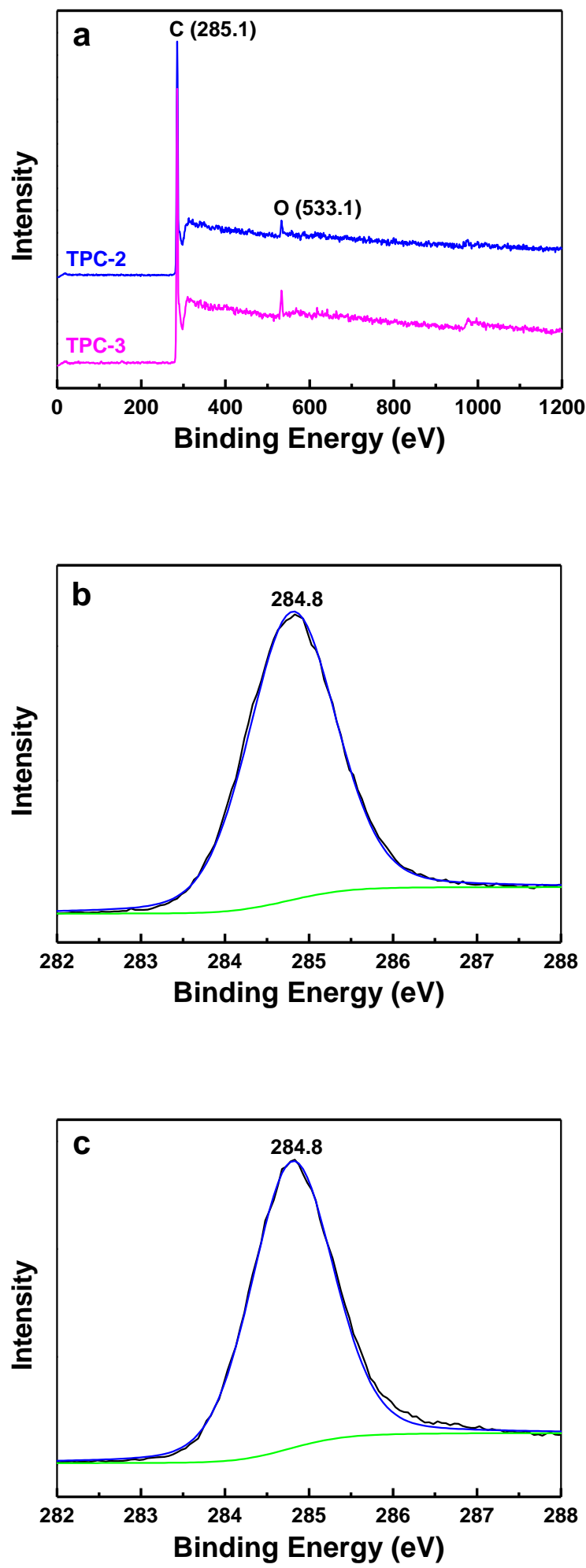

Figure 2 

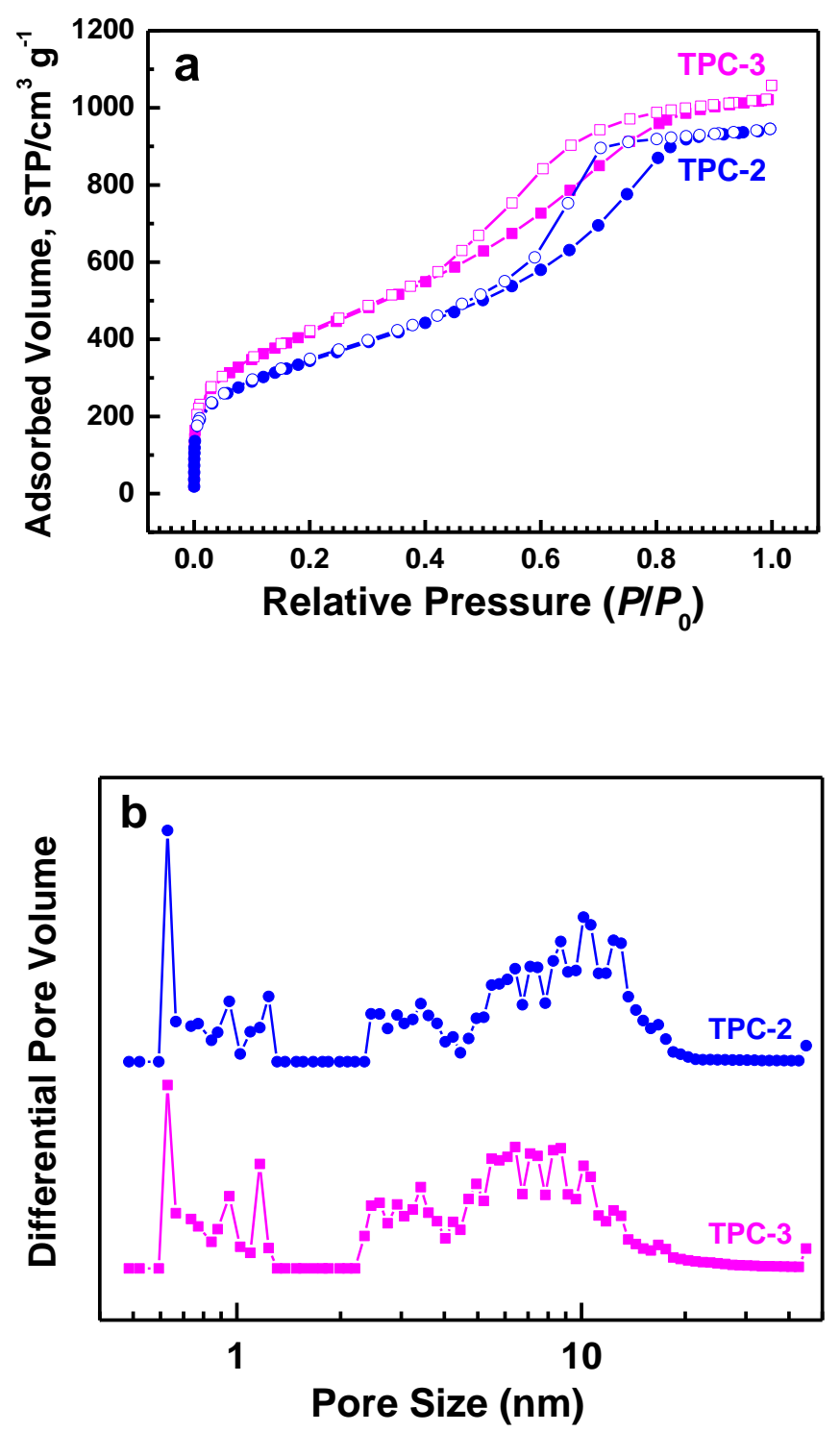

Figure 3 


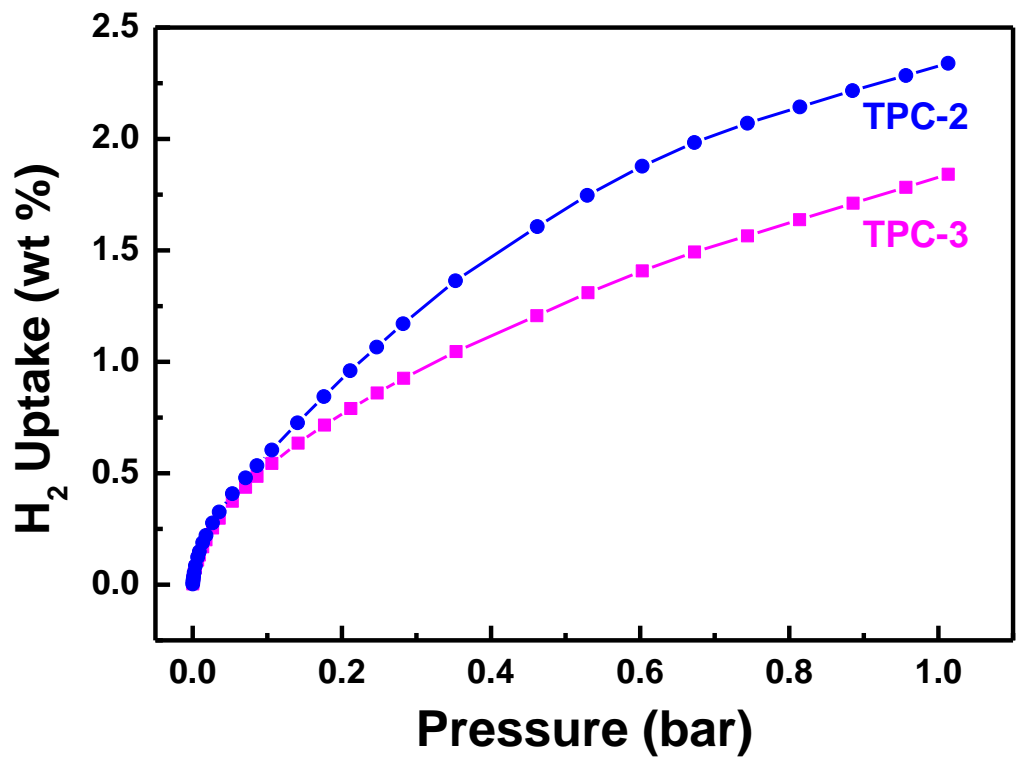

Figure 4 


\section{Graphic Abstract}

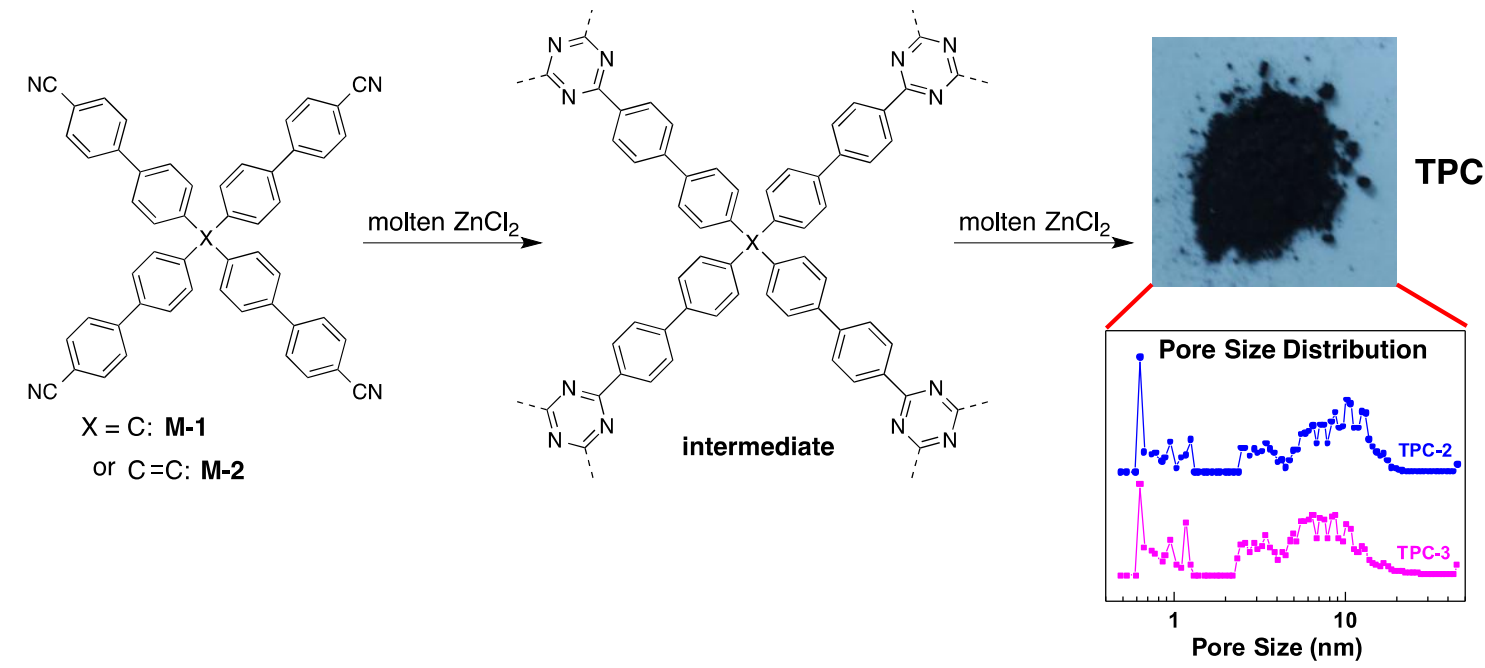


